\title{
Metabostemness: Metaboloepigenetic reprogramming of cancer stem-cell functions
}

\author{
Javier A. Menendez ${ }^{1,2}$, Bruna Corominas-Faja ${ }^{1,2}$, Elisabet Cuyàs ${ }^{1,2}$ and Tomás \\ Alarcón ${ }^{3,4}$ \\ ${ }^{1}$ Metabolism \& Cancer Group, Translational Research Laboratory, Catalan Institute of Oncology (ICO), Girona, Catalonia, \\ Spain \\ ${ }^{2}$ Girona Biomedical Research Institute (IDIBGI), Girona, Catalonia, Spain \\ ${ }^{3}$ Computational \& Mathematical Biology Research Group, Centre de Recerca Matemàtica (CRM), Barcelona, Catalonia, Spain \\ ${ }^{4}$ Departament de Matemàtiques, Universitat Autònoma de Barcelona (UAB), Barcelona, Catalonia, Spain \\ Correspondence to: Javier A. Menendez, email: jmenendez@iconcologia.net
}

Keywords: Oncometabolites, reprogramming, stemness, cancer, stem cells, cancer stem cells

Received: December 16, $2014 \quad$ Accepted: December 22, 2014

Published: December 26, 2014

This is an open-access article distributed under the terms of the Creative Commons Attribution License, which permits unrestricted use, distribution, and reproduction in any medium, provided the original author and source are credited.

\section{ABSTRACT}

\begin{abstract}
Cancer researchers are currently embarking on one of their field's biggest challenges, namely the understanding of how cellular metabolism or certain classes of elite metabolites (e.g., oncometabolites) can directly influence chromatin structure and the functioning of epi-transcriptional circuits to causally drive tumour formation. We here propose that refining the inherent cell attractor nature of nuclear reprogramming phenomena by adding the under-appreciated capacity of metabolism to naturally reshape the Waddingtonian landscape's topography provides a new integrative metabolo-epigenetic model of the cancer stem cell (CSC) theory.
\end{abstract}

By emphatically underscoring the similarities in nuclear reprogramming pathways involved in the generation of induced pluripotent stem cells (iPSCs) and cancer stem cells (CSCs), one of the conclusions drawn from the 2013 Nature-Ludwig Institute for Cancer Research Conference on "Nuclear Reprogramming and the Cancer Genome" has been to suggest a new cancer hallmark, namely mutations or expression changes in metabolic genes that are implicated in the regulation of DNA methylation plasticity [1].

Oncometabolites and the stem cell origin of cancers: A Darwinian view

In cancers with a stem cell origin such as haematopoietic malignancies, gain-of-function isocitrate dehydrogenases (IDH) mutations generating the oncometabolite 2-hydroxyglutarate (2HG) lead to global hypermethylation [2]. This activity prevents the demethylation of genes that are implicated in differentiation, consequently promoting a stabilization of undifferentiated and self-renewing cellular states that may be targetable and expanded by later transforming mutations. For most solid tumours with non-stem cell origins, including liver, breast, lung, pancreatic and prostate cancer, in which mutations in metabolic genes are not widespread, it was alternatively proposed that an intact metabolic function of IDH would be necessary to maintain DNA methylation plasticity and a flexible epigenetic landscape [1].

The latter proposal, however, can be somewhat difficult to reconcile with the actual functioning of the oncometabolite $2 \mathrm{HG}$ in solid tumours such as intrahepatic cholangiocarcinomas (IHCCs) [3] and breast carcinomas (BCs) [4]. IHCC is a deadly liver malignancy in which highly prevalent $2 \mathrm{HG}$-producing IDH mutations subvert the hepatocyte differentiation/quiescence program to create a persistent pre-neoplastic state, which is primed for transformation into adenocarcinoma by additional oncogenic mutations [3]. In the absence of IDH mutations, the accumulation of $2 \mathrm{HG}$ is part of the $c$ - $M y c$-driven metabolic reprogramming observed in biologically aggressive BCs that exhibit globally increased DNA methylation [4]. The hypermethylation phenotype of $2 \mathrm{HG}-$ overexpressing IHCC and BC is characterised by a strong enrichment of a stem cell-like transcriptional signature [3, 4]. Why do oncometabolic traits convergently "encode" an 
A
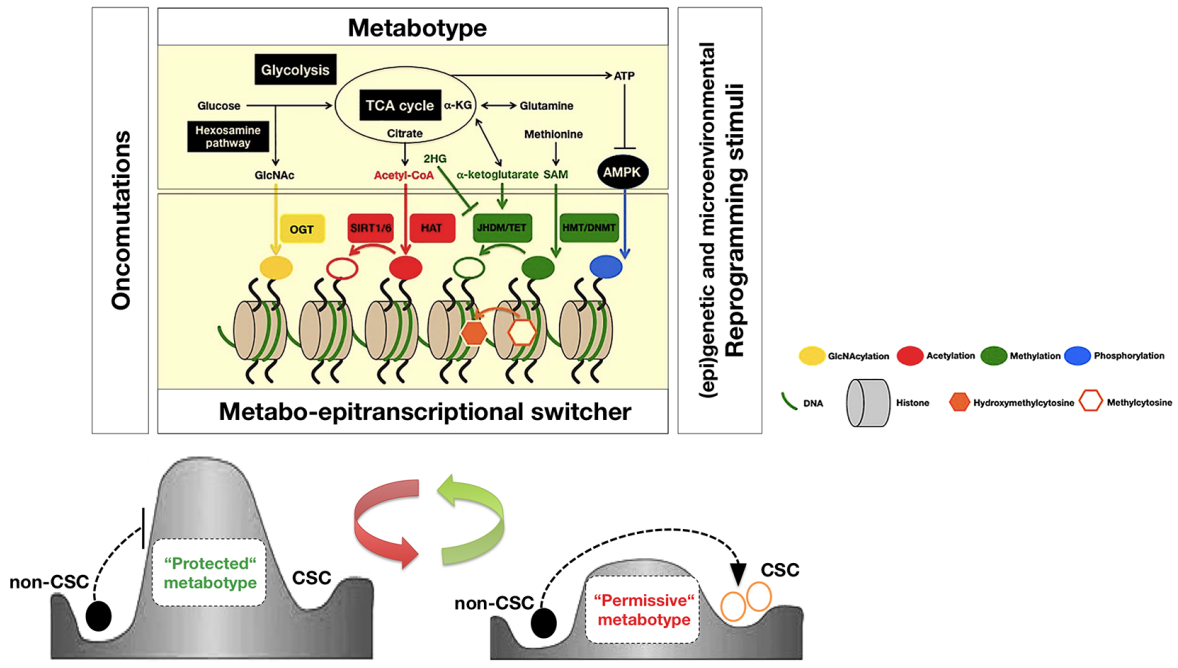

B

\section{Cancer with a stem cell of origin}

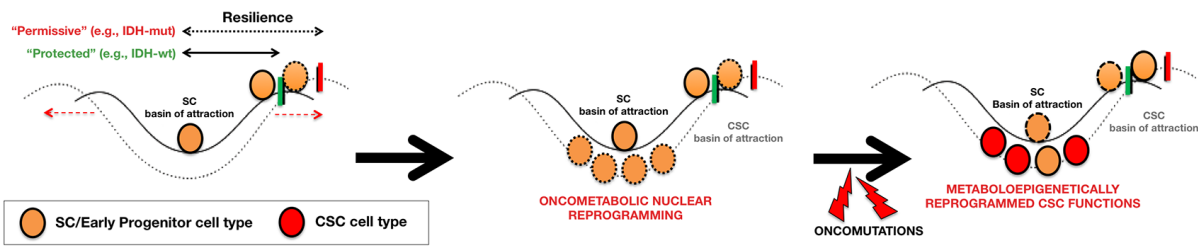

\section{Cancer with non-stem cell of origin}

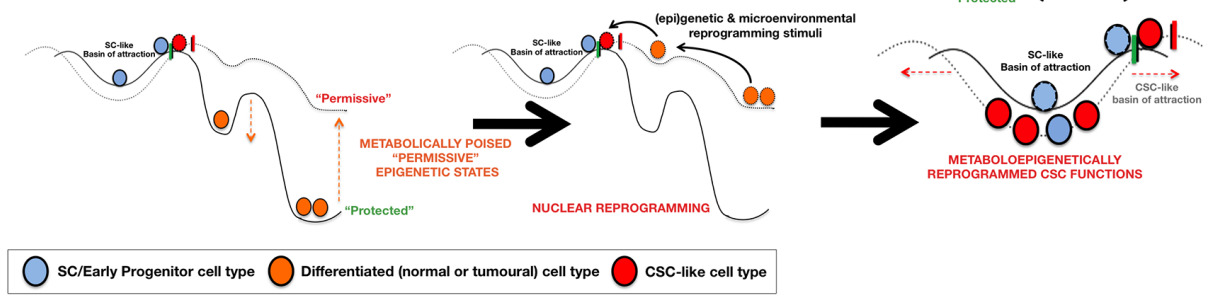

Figure 1: A Waddingtonian perspective of the metabolo-epigenetic reprogramming of stemness in cancer tissues. A. A highly active crosstalk process between certain metabotypic features, elite metabolites (e.g., oncometabolites), and epigenetics could allow the causal integration of the metabolism with genetic programs to generate CSC functions via pathological nuclear reprogramming $[1,9]$. The acquisition of stemness in cancer tissues might not only be hard-wired by the mutational landscape but also by the pivotal regulatory role of the cellular metabotype. This relationship, in turn, can remove, diminish, or modify the nature of the molecular barriers present in Waddington's epigenetic landscapes, thus allowing cells to more easily (re-)enter into CSC cellular macrostates. In this metabostemness framework, even modest changes in the "protected" versus "permissive" nature of the cellular metabotype are expected to produce a considerable change in the global kinetic efficiency of the CSC reprogramming process. The cellular metabotype can be described by means of variability criteria, such as the presence or absence of particular metabolites (e.g., oncometabolites such as $2 \mathrm{HG}$ ), the concentration levels of certain metabolites, the relative levels or ratios between specific metabolites, metabolic profiles or even spatio-temporal flux distributions of metabolites (e.g., N-acetylglucosamine [GlcNAc] for histone GlcNAcylation, the $\mathrm{NAD}^{+} / \mathrm{NADH}$ ratio for sirtuin histone deacetylase activities, acetyl-CoA as a donor for histone acetylation, alpha-ketoglutarate as a cofactor for histone and DNA demethylation reactions, S-adenosylmethionine $[\mathrm{SAM}]$ as a donor for DNA methylation, or ATP/AMP-regulated chromatin translocation of AMPK for histone phosphorylation). B. The cellular metabotype may act as "starter dough" that renders any type of cell-of-origin susceptible to the epigenetic rewiring required for the acquisition of refractoriness to differentiation [9]. This modification can significantly alter the efficiency and speed of CSC reprogramming in cancers with (top) and without (bottom) a stem cell origin by lowering the barriers of the epigenetic landscape and increasing the size of the basins of attraction, which are necessarily located in the developmentally immature, stem-like regions of the "higher mountains" of the landscape. From a therapeutic perspective, small perturbations in a particular metabolic pathway or metabolite might have drastic consequences on the formation, maintenance, and evolution of CSC cellular states. Indeed, the unexpected applications for biguanides in oncology might closely relate to their metabolic effects during the induction of CSCs [15-17]. (OGT: O-linked N-acetylglucosamine transferase; SIRT1/6: NAD+-dependent SIRTUIN histone deacetylases (HDACs); HAT: Histone acetyltransferases; JHDM/TET: Jumonji-C domain containing histone demethylases (HDMs)/Ten-eleven translocation (Tet) methylcytosine dioxygenases; HMT/DNMT: Histone methyltransferase/DNA methyltransferases) 
immature, stem-like program in cancer tissue, regardless of the stem cell/non-stem cell source?

It has been argued that the cell-of-origin dictates the metaboloepigenetic relationship between nuclear reprogramming and the generation of CSCs [1], an ad hoc evolutionary assumption in which natural forces might select for either "hierarchic" or "dynamic" epigenetic landscapes depending on their stem cell or non-stem cell origin, respectively. Instead, we here propose that refining the inherent cell attractor nature of nuclear reprogramming phenomena [5-8] by adding the under-appreciated capacity of metabolism to naturally reshape the Waddingtonian landscape's topography provides a new integrative perspective of the CSC theory that is free of any ad hoc argumentation.

\section{Metaboloepigenetic reprogramming of cancer stem cells: A Waddingtonian view}

We recently coined the term "metabostemness" to refer to the metabolic parameters causally controlling or functionally substituting the epi-transcriptional orchestration of CSC nuclear reprogramming [9]. A central metabostemness element is the metabolo-epitranscriptional switcher, which decodes the metabolism's ability to interfere with the developmental Waddington's "buffering" and "canalization" via the removal, lowering or modification of landscape "energy" barriers (Fig. 1A). Two key operational features determine the functioning of metabostemness: a.) Two primary epigenetic codes, DNA methylation and histone modification, and the consequent epigenetic regulation of cell differentiation genes are the pivotal molecular events that account for the regulatory effects of metabolism on nuclear reprogramming [10], and b.) the switcher is responsive not only to bona fide oncometabolites but also to more common primary metabolites employed by chromatin-remodelling enzymes [9, 10], implying that even small changes in the landscape's fine topography (e.g., metabolically driven leaning of a slope in a separating barrier) could sufficiently place cells into the basins of CSC attractors without the intervention of mutational events. In cancers with a stem cell origin, certain metabotypes might provoke cells to get stuck very near or in the same state-space of attractors of previously normal stem cells (e.g., by increasing the size of the basins and, therefore, the resilience of stem-like states), which will subsequently increase the probability of undifferentiated cells targetable by pro-proliferative oncomutations (Fig. 1B, top). For cancers with nonstem cell origins, certain metabotypes can permissively alleviate the "uphill", unfavourable developmental process of "jumping back" from non-CSC differentiated valleys to high-altitude CSC attractors while concomitantly promoting the ground-state character of self-maintaining CSC-like states (Fig. 1B, bottom).

Cell types can occupy attractors even after the metabolic stimulus triggering the transition disappears, a "memory effect" in a multi-attractor, flexible landscape that explains why the transient occurrence of CSCpromoting events (e.g., hypoxia- or acidic pH-induced loss of pro-differentiation factors) $[11,12]$ are sufficient to generate a lasting CSC population (e.g., long-lived dormant stem cell-like cells). If certain metabotypes confer a proliferative advantage on cells trapped in abnormal attractors, somatic evolution can further deepen them and, therefore, promote their over-occupancy by specific cancer cell types (e.g., IDH-mutated IHCC with stem cell features and exhibiting bile duct differentiation) [3].

\section{CONCLUSIONS}

CSC states can be viewed as inherently inevitable epigenetic deviations of Waddington's developmental landscapes in which metabolism modifies the probability that not only stem cells/early progenitors but also normal or tumour-differentiated cells can find either pre-existing or de novo occupied, self-organising attractors encoding dynamically robust CSC signatures that somatic evolution alone cannot explain. Oncometabolites can therefore operate as the metabolic basis for the epigenetic landscape of tumour-initiating events regardless of the stem cell/nonstem cell source and without the requirement of mutations in metabolic enzymes $[13,14]$.

\section{ACKNOWLEDGMENTS}

This work was supported by grants from the Ministerio de Ciencia e Innovación (Grant SAF201238914), Plan Nacional de I+D+I, Spain and the Agència de Gestió d'Ajuts Universitaris I de Recerca (AGAUR) (Grant 2014 SGR229), Departament d'Economia I Coneixement, Catalonia, Spain.

\section{CONFLICT OF INTEREST STATEMENT}

The authors of this manuscript have no conflicts of interest to declare.

\section{REFERENCES}

1. Goding CR, Pei D, Lu X. Cancer: pathological nuclear reprogramming? Nat Rev Cancer. 2014; 14: 568-573.

2. Yang M, Soga T, Pollard PJ. Oncometabolites: linking altered metabolism with cancer. J Clin Invest. 2013; 123: 3652-3658.

3. Saha SK, Parachoniak CA, Ghanta KS, Fitamant J, Ross KN, Najem MS, Gurumurthy S, Akbay EA, Sia D, Cornella H, Miltiadous O, Walesky C, Deshpande V, Zhu AX, Hezel AF, Yen KE, Straley KS, Travins J, Popovici-Muller J, Gliser C, Ferrone CR, Apte U, Llovet JM, Wong KK, Ramaswamy S, Bardeesy N. Mutant IDH inhibits HNF- 
4a to block hepatocyte differentiation and promote biliary cancer. Nature. 2014; 513:110-114.

4. Terunuma A, Putluri N, Mishra P, Mathé EA, Dorsey TH, Yi M, Wallace TA, Issaq HJ, Zhou M, Killian JK, Stevenson HS, Karoly ED, Chan K, Samanta S, Prieto D, Hsu TY, Kurley SJ, Putluri V, Sonavane R, Edelman DC, Wulff J, Starks AM, Yang Y, Kittles RA, Yfantis HG, Lee DH, Ioffe OB, Schiff R, Stephens RM, Meltzer PS, Veenstra TD, Westbrook TF, Sreekumar A, Ambs S. MYC-driven accumulation of 2-hydroxyglutarate is associated with breast cancer prognosis. J Clin Invest. 2014; 124: 398-412.

5. Huang S. Reprogramming cell fates: reconciling rarity with robustness. Bioessays. 2009; 31: 546-560.

6. Huang S, Ernberg I, Kauffman S. Cancer attractors: a systems view of tumors from a gene network dynamics and developmental perspective. Semin Cell Dev Biol. 2009; 20: 869-876.

7. Hanna JH, Saha K, Jaenisch R. Pluripotency and cellular reprogramming: facts, hypotheses, unresolved issues. Cell. 2010; 143: 508-525.

8. Huang S. The molecular and mathematical basis of Waddington's epigenetic landscape: a framework for postDarwinian biology? Bioessays. 2012; 34: 149-157.

9. Menendez JA, Alarcón T. Metabostemness: A new cancer hallmark. Front Oncol. 2014: 4: 262.

10. $\mathrm{Lu} \mathrm{C}$, Thompson $\mathrm{CB}$. Metabolic regulation of epigenetics. Cell Metab. 2012; 16: 9-17.

11. Mariani CJ, Vasanthakumar A, Madzo J, Yesilkanal A, Bhagat T, Yu Y, Bhattacharyya S, Wenger RH, Cohn SL, Nanduri J, Verma A, Prabhakar NR, Godley LA. TET1mediated hydroxymethylation facilitates hypoxic gene induction in neuroblastoma. Cell Rep. 2014; 7: 1343-1352.

12. Hjelmeland AB, Wu Q, Heddleston JM, Choudhary GS, MacSwords J, Lathia JD, McLendon R, Lindner D, Sloan A, Rich JN. Acidic stress promotes a glioma stem cell phenotype. Cell Death Differ. 2011; 18: 829-840.

13. Shim EH, Livi CB, Rakheja D, Tan J, Benson D, Parekh V, Kho EY, Ghosh AP, Kirkman R, Velu S, Dutta S, Chenna B, Rea SL, Mishur RJ, Li Q, Johnson-Pais TL, Guo L, Bae S, Wei S, Block K, Sudarshan S. 1-2-Hydroxyglutarate: An Epigenetic Modifier and Putative Oncometabolite in Renal Cancer. Cancer Discov. 2014; 4: 1290-1298.

14. Nam H, Campodonico M, Bordbar A, Hyduke DR, Kim S, Zielinski DC3, Palsson BO5. A Systems Approach to Predict Oncometabolites via Context-Specific GenomeScale Metabolic Networks. PLoS Comput Biol. 2014; 10, e1003837.

15. Pollak M. Potential applications for biguanides in oncology. J Clin Invest. 2013; 123: 3693-3700.

16. Hirsch HA, Iliopoulos D, Struhl K. Metformin inhibits the inflammatory response associated with cellular transformation and cancer stem cell growth. Proc Natl Acad Sci USA. 2013; 110: 972-977.

17. Janzer A, German NJ, Gonzalez-Herrera KN, Asara JM,
Haigis MC, Struhl K. Metformin and phenformin deplete tricarboxylic acid cycle and glycolytic intermediates during cell transformation and NTPs in cancer stem cells. Proc Natl Acad Sci USA. 2014; 111: 10574-10579. 